Proc. Indian Acad. Sci.' (Chem. Sci.), Vol. 104, No. 3, June 1992, pp. 399-407.

(C) Printed in India.

\title{
Molecular interpretation of the linear relationship between the entropy and the enthalpy of activation of charge transfer reactions in polar liquids
}

\author{
A CHANDRA ${ }^{\dagger}$ and B BAGCHI* \\ Solid State and Structural Chemistry Unit, Indian Institute of Science, Bangalore 560012 , \\ India \\ ${ }^{\dagger}$ Present address: Department of Chemistry, University of British Columbia, Vancouver, \\ BC V6T 1Z1, Canada \\ MS received 6 September 1991

\begin{abstract}
The well-known linear relationship $\left(T \Delta S^{*}=\alpha \Delta H^{\#}+\beta\right.$, where $\left.1>\alpha>0, \beta>0\right)$ between the entropy $\left(\Delta S^{\#}\right)$ and the enthalpy $\left(\Delta H^{*}\right)$ of activation for reactions in polar liquids is investigated by using a molecular theory. An explicit derivation of this linear relation from first principles is presented for an outersphere charge transfer reaction. The derivation offers microscopic interpretation for the quantities $\alpha$ and $\beta$. It has also been possible to make connection with and justify the arguments of Bell put forward many years ago.
\end{abstract}

Keywords. Molecular interpretation; linear relationship; entropy; enthalpy of activation; polar liquids; charge transfer reactions.

\section{Introduction}

The study of the effects of solvent on chemical reactions in solution has been a subject of great importance in chemistry (Glasstone et al 1941; Amis 1966; Entelis and Tiger 1976; Reichardt 1979). It was recognized quite early that the solvent can significantly affect the reaction potential energy surface, espectially if the reaction involves polar species in a dipolar liquid which is most often the case. The following expression for the rate constant, $k$,

$$
k=\left(k_{B} T / h\right) \exp \left(\Delta S^{\#} / k_{B}\right) \exp \left(-\Delta H^{*} / k_{B} T\right)
$$

has been used widely to rationalize the solvent effects on chemical reactions (Wynne-Jones and Eyring 1935). In (1), $\Delta S^{*}$ and $\Delta H^{*}$ are the entropy and the enthalpy of activation per molecule, respectively, $k_{B}$ is the Boltzmann constant, $h$ is Planck's constant and $T$ is the temperature.

Recently great attention has been focussed on understanding the dynamic solvent effects on various ultra-fast chemical reactions (Hynes 1985; Kosower and Huppert 1986; Barbara and Jarzeba 1988, 1990; Simon 1988; Bagchi 1989; Marroncelli et al 1989; Fleming and Wolynes 1990; Bagchi and Chandra 1991) and a better understanding of both the equilibrium and the dynamic solvent effects is beginning to emerge. Several

\footnotetext{
* For correspondence
} 
microscopic studies are now available which can be used to address some of the long-standing problems in chemical dynamics.

One such problem is the often observed linear relationship between the enthalpy and the entropy of activation given by (Evans and Polanyi 1936; Bell 1937; Amis 1966)

$$
T \Delta S^{*}=\alpha \Delta H^{*}+\beta .
$$

It is known experimentally that $\alpha$ is positive and less than unity. The intercept $\beta$ also appears to be positive (Evans and Polanyi 1936; Bell 1937; Amis 1966). Equation 2 is interesting (and also important) for the following reasons. Firstly, if solvent effects are not important, than there is no a priori reason that (2) should hold and no such constraint seems possible. If solvent effects are important, then such an equation is possible because both $\Delta H^{*}$ and $\Delta S^{*}$ may partly have the same origin. Even then (2) is non-trivial because $\Delta S^{\#}$ and $\Delta H^{*}$ are rather different thermodynamic quantities. Secondly, if a relation like (2) is truly valid, then it would have serious implications for the experimental determination of the activation energy of the reaction. For example, if $\beta$ is small, then a determination of the enthalpy of activation by the usual temperature variation method would actually measure $(1-\alpha) \Delta H^{*}$, instead of $\Delta H^{*}$.

There have been attempts in the past to explain (2). Evans and Polanyi (1936) noted that a relation of the form of (2) also holds for enthalpy and entropy of solution of the same solute in a series of different solvents

$$
T \Delta S=\alpha \Delta H+\beta,
$$

where $\Delta S$ and $\Delta H$ now refer to the entropy and the enthalpy of solution. Therefore, if one assumes that the equilibrium relation (3), holds, not only for the reactant, but also for the activated complex, then one naturally recovers (2).

Many years ago Bell (1937) investigated the reason for the existence of a linear relation between $T \Delta S$ and $\Delta H$ by using the basic statistical thermodynamics. He arrived at the conclusion that a relation like (3) is possible only when the interaction between the solute and the solvent molecules is small and also. when the solute molecule is considerably smaller than the solvent molecules. The argument of Bell was based on qualitative grounds. It fails to account satisfactorily, as recognized by Bell himself, for the relation given by (2) and (3) which exist for a large solute in different solvents.

A limited explanation for these relations can be given for the solvation of a large dipolar solute in a dipolar liquid, if one assumes that the main source of $\Delta H$ and $\Delta S$ is the electrical interaction between the solute and the solvent and also if the solvent can be replaced by a dielectric continuum. Under these assumptions, the free energy of solute-solvent interaction can be given by (Onsager 1935; Frohlich 1958)

$$
\Delta A=-\boldsymbol{\mu} \cdot \mathbf{R}+\gamma R^{2},
$$

where $\boldsymbol{\mu}$ is the dipole moment of the solute and $\mathbf{R}$ is the reaction field at the solute cavity. If one minimizes the free energy with respect to $\mathbf{R}$, then one obtains

$$
\Delta A=-\frac{1}{2} \boldsymbol{\mu} \cdot \mathbf{R} .
$$

Under the same conditions the change in the energy of the system $(\Delta E)$ and the change in the enthalpy $(\Delta H)$ are both equal to $-\boldsymbol{\mu} \cdot \mathbf{R}$. Next, the use of the thermodynamic relation $\Delta A=\Delta H-T \Delta S$ gives the following relation between $\Delta H$ and $T \Delta S$,

$$
T \Delta S=\frac{1}{2} \Delta H
$$


which is a particular form of (3) with $\alpha=0.5$ and $\beta=0$. However, one knows experimentally that $\beta \neq 0$. Also, the above derivation, given originally by Frohlich (1958) in a different context, is severely limited.

In the following we present a molecular derivation of (2) and (3). Towards this end, we first note that the main effect of the solute-solvent interaction is to produce an inhomogeneity in the density distribution (primarily an orientational polarization) near the solute - as recognized by Bell (1937). However, Bell could not carry out a microscopic calculation because the necessary statistical thermodynamic expressions for the free energy and the distribution functions were not available at that time. We next discuss our theoretical treatment.

\section{Theory}

For simplicity and also for making the calculations precise, we consider a model electron transfer reaction in a dipolar liquid. However, the treatment that follows is quite general and can be used to extract a functional relation between $\Delta H$ and $\Delta S$ for other reactions also. The electron transfer that we study involves a redox couple of the following type

$$
\mathrm{Ox}+e \rightleftharpoons \text { Red. }
$$

This model has been studied recently by McMannis and Weaver (1989) to investigate the dynamic solvent effects on electron transfer reactions. As pointed out by McMannis and Weaver (1989), this reaction can be thought of as an electrochemical exchange process with the reactant located sufficiently far from the metal surface. The free energy of activation, $\Delta A^{*}$, is the intrinsic outersphere (i.e., the solvent) part of the barrier energy. Thus, for this model of electron transfer reaction, the free energy of activation is equal to the solvation energy of an $e / 2$ amount of charge where $e$ is the charge on an electron (Marcus 1956, 1964; McMannis and Weaver 1989). An accurate expression of the solvation energy of a solute can be derived from the density functional theory (DFT) (Bagchi and Chandra 1989; Chandra and Bagchi 1989b, e, 1990). As we have mentioned before, the charge distribution of the solute or the reacting system creates an inhomogeneous polarization in the solvent. This polarization is related to the position and orientation dependent solvent density. The DFT provides an expression for the free energy change of the total system because of the inhomogeneity in the solvent density distribution which is caused by the reactant system. For charge transfer reactions the relevant polarization is the longitudinal one. The DFT can be used to obtain a general microscopic expression for the polarization dependent free energy functional in presence of an external electric field $\mathbf{E}_{\mathbf{0}}(\mathbf{k})$. This expression is given by the following equation (Chandra and Bagchi 1989d, 1991)

$$
\begin{aligned}
A\{P(k)\}= & A_{0}+\int \mathrm{d} \mathbf{k} P^{2}(\mathbf{k})-\frac{1}{3 y(2 \pi)^{2}} \frac{\rho_{0}}{4 \pi} \int \mathrm{dk} P(\mathbf{k}) C_{110}(k) P(-\mathbf{k}) \\
& -(2 \pi)^{-3} \int \mathrm{d} \mathbf{k} P(k) E_{0}(k),
\end{aligned}
$$

where $A_{0}$ is the free energy of the homogeneous unperturbed liquid. $P(k)$ is the 
inhomogeneous longitudinal polarization of the liquid and $C_{110}(k)$ is the (110) component of the two-particle direct correlation function of the liquid in its spherical harmonic expansion. $Y$ is equal to $\left(4 \pi \rho_{0} \mu^{2} / 9 k_{B} T\right)$ where $\rho_{0}$ is the number density of the liquid. The derivation of the above equation has been discussed in detail by Chandra and Bagchi $(1989,1990)$. The equilibrium value of the polarization, $P(k)$, can be obtained by minimizing the free energy with respect to the polarization. The expression of the equilibrium polarization is given by the following equation

$$
P(k)=\frac{3 Y}{4 \pi} S_{110}(k) E_{0}(k),
$$

where $S_{110}(k)$ is the (110) component of the static structure factor of the liquid which is related in a linear theory to the direct correlation function coefficient $C_{110}(k)$ by the following relation (Grey and Gubbins 1984; Hansen and McDonald 1986)

$$
S_{110}(k)=\left[1-\frac{\rho_{0}}{4 \pi} C_{110}(k)\right]^{-1} .
$$

Now we substitute (9) in (8) to obtain the following expression of the free energy of activation $\Delta A^{*}$

$$
\Delta A^{*}=-\frac{1}{8 \pi}(2 \pi)^{-3} 3 Y \int \mathrm{dk} S_{110}(k) E_{0}^{2}(k),
$$

where $E_{0}(k)$ is the wavevector dependent electric field of the reactant with $e / 2$ amount of charge. The static structure factor, $S_{110}(k)$, can be related to the wavevector dependent static dielectric function of the liquid (Neuman 1986; Chandra and Bagchi 1989a, c; Wei and Patey 1990; Raineri et al 1991). Our next step is to calculate the entropy and the enthalpy of activation. We note that at constant volume, $\Delta S$ and $\Delta A$ are related by the following thermodynamic relation

$$
\Delta S=-\left.\frac{\partial}{\partial T} \Delta A\right|_{0} .
$$

We take the temperature derivative of (11) to obtain the following expression for $\Delta S^{\prime \prime}$

$$
\begin{aligned}
T \Delta S^{*}=- & \frac{1}{8 \pi}(2 \pi)^{-3} 3 Y \int \mathrm{d} \mathbf{k} S_{110}(k) E_{0}^{2}(k) \\
& +\frac{1}{8 \pi}(2 \pi)^{-3} T 3 Y \int \mathrm{d} \mathbf{k} E_{0}^{2}(k) \frac{\partial}{\partial T} S_{110}(k) .
\end{aligned}
$$

At constant pressure and volume the change in internal energy $(\Delta E)$ and the change in enthalpy $(\Delta H)$ are equal to each other. Thus, we obtain

$$
\begin{aligned}
\Delta H^{\#}= & \Delta A^{\#}+T \Delta S^{\#} \\
=- & \frac{1}{4 \pi}(2 \pi)^{-3} 3 Y \int \mathrm{dk} S_{110}(k) E_{0}^{2}(k) \\
& +\frac{1}{8 \pi}(2 \pi)^{-3} T 3 Y \int \mathrm{dk} E_{0}^{2}(k) \frac{\partial}{\partial T} S_{110}(k) .
\end{aligned}
$$


Equations (13) and (14) can now be used to obtain the following exact relation between $\Delta S^{*}$ and $\Delta H^{\#}$

where

$$
T \Delta S^{\#}=\alpha \Delta H^{\#}+\beta,
$$

$$
\begin{aligned}
& \alpha=0.5 \\
& \beta=\frac{1}{16 \pi}(2 \pi)^{-3} T 3 Y \int \mathrm{dk} E_{0}^{2}(k) \frac{\partial}{\partial T} S_{110}(k) .
\end{aligned}
$$

The above equation is the main result of this paper. Several comments on this are in order.

(1) Equation (14) gives a definite constant value of $\alpha$ which is equal to 0.5 . Thus, $\alpha$ is independent of both the solute and the solvent. The predicted value of $\alpha$ is in moderate agreement with many experimental results (Evans and Polanyi 1936; Bell 1937; Amis 1966).

(2) The intercept $\beta$ depends on the temperature derivative of the static structure of the solvent. Thus $\beta$ is a solvent dependent quantity. It also depends on the size of the solute (through $E_{0}(k)$ ). Thus, if one varies the solvent, keeping the solute fixed, or vice-versa, the value of the quantity $\beta$ can change in both the cases. Therefore, strictly speaking, a general relation of the form (2) between $\Delta S^{*}$ and $\Delta H^{*}$ with a constant $\beta$ is not obvious from (15). For dipolar liquids, the change of orientational structure with temperature may not differ substantially from solvent to solvent and we may expect a near linear relation between $\Delta S^{*}$ and $\Delta H^{*}$ for a given reacting system in different solvents. Numerical calculations confirm this prediction, as discussed in the next section. The case of different solutes in the same solvent is more complex and is also discussed later.

(3) We have considered the case of an outer sphere electron transfer reaction. It is obvious that the same formulation holds for the process of solvation of charged solutes in polar solvents. Thus, all the above conclusions are valid for the relationship between the entropy and the enthalpy of solvation in polar solvents.

\section{Numerical results}

We have explored numerically the validity of (2) for the following two cases: (i) the reacting system remains the same and the solvent is varied, (ii) the size of the reacting system is varied while the solvent remains the same. We have used the mean spherical approximation (MSA) (Wertheim 1971) to calculate the static structure factor of the liquid and its temperature derivative. The reason for using MSA is that it provides a simple analytic solution for the structure factor which is fairly accurate for not very strongly polar liquids.

In figure 1 we have shown the variation of $\beta$ with the dielectric constant of the solvent. The sizes of the solute and the solvent molecules are assumed to be the same. It is seen that $\beta$ is nearly constant for the entire range of the dielectric constant that is investigated. Thus, a linear relation between $T \Delta S^{*}$ and $\Delta H^{*}$ holds in this case which is shown in figure 2. As we have mentioned earlier, such a linear relation between $T \Delta S^{\#}$ and $\Delta H^{\#}$ was observed by Evans and Polanyi (1936) for solvation in different solvents. 


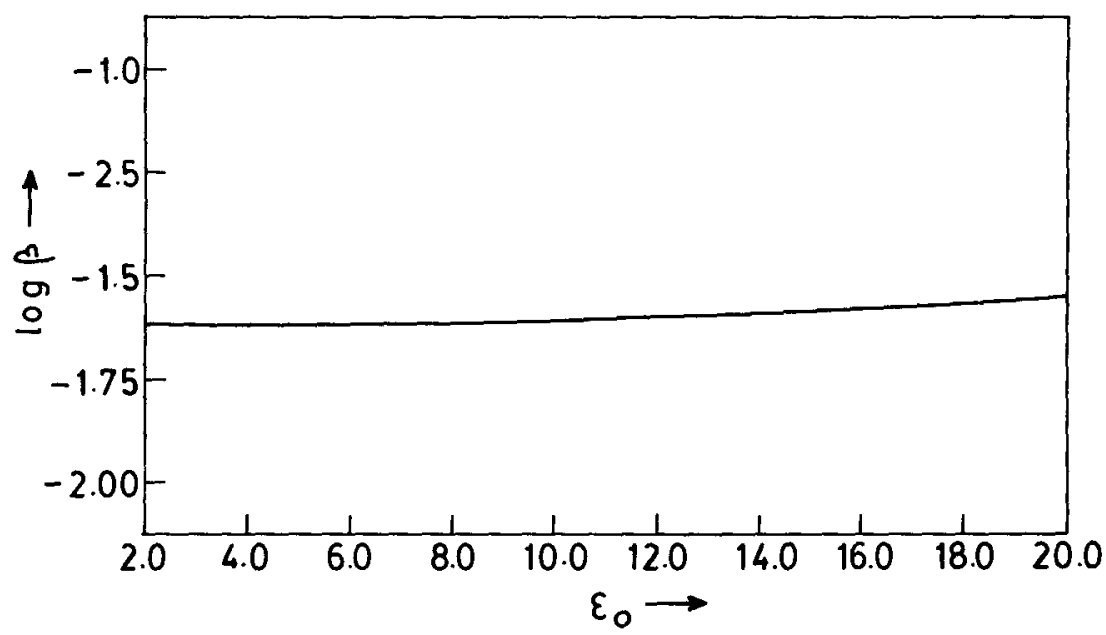

Figure 1. The dependence of $\beta,(15)$, on the dielectric constant of the solvent. $\beta$ is calculated in units of $e^{2} / \sigma$ where $e$ and $\sigma$ are the charge of an electron and the diameter of a solvent molecule, respectively. The ratio of the reactant-solvent molecular size ratio, $R$, is $1 \cdot 0$. The value of the reduced density of the solvent, $\rho_{0} \sigma^{3}$, is $0-8$.

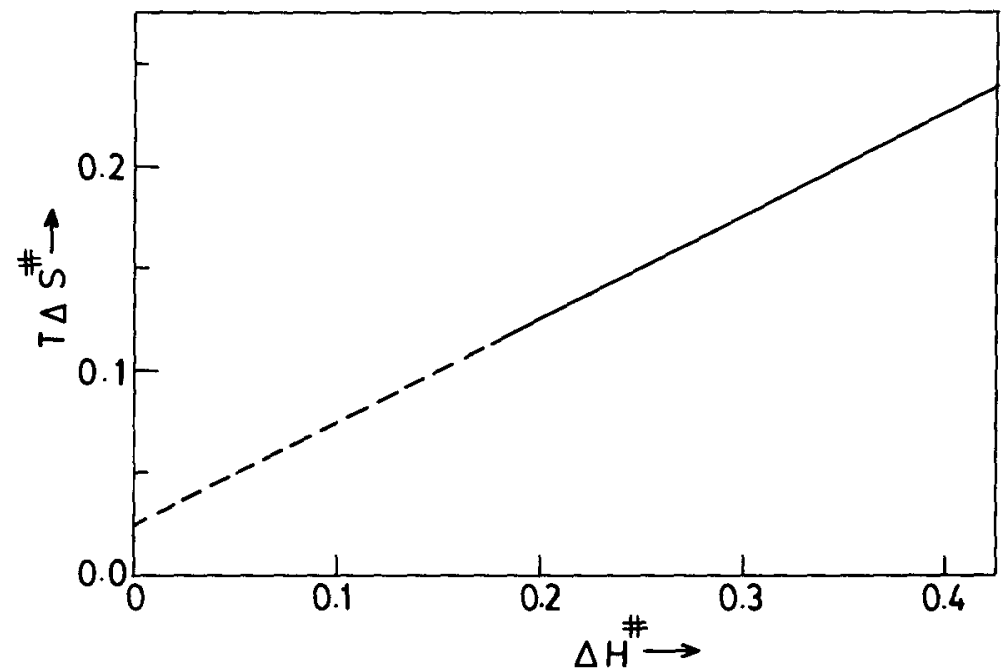

Figure 2. The plot of entropy of activation ( $\left.T \Delta S^{\#}\right)$ against the enthalpy of activation $\left(\Delta H^{*}\right)$ calculated for different values of the dielectric constant of the medium. The solid curve shows the calculated values and the dashed one is the extrapolation to the zero enthalpy change. The values of the other parameters are the same as in figure 1.

Figure 3 shows the variation of $\beta$ with the size of the reacting system. The dielectric constant of the solvent is kept fixed at $18 \cdot 0 . R$ is the reactant-solvent molecular size ratio. It is seen that for very small solutes, $\beta$ is nearly constant. $\beta$ decreases smoothly as the size of the solute is increased. It is clear that the linear relation between $T \Delta S^{*}$ and $\Delta H^{\#}$ exists in this case only for very small solutes which is in agreement with the conjecture of Bell (1937). In the opposite limit, that is for very large reactants, $\beta$ 


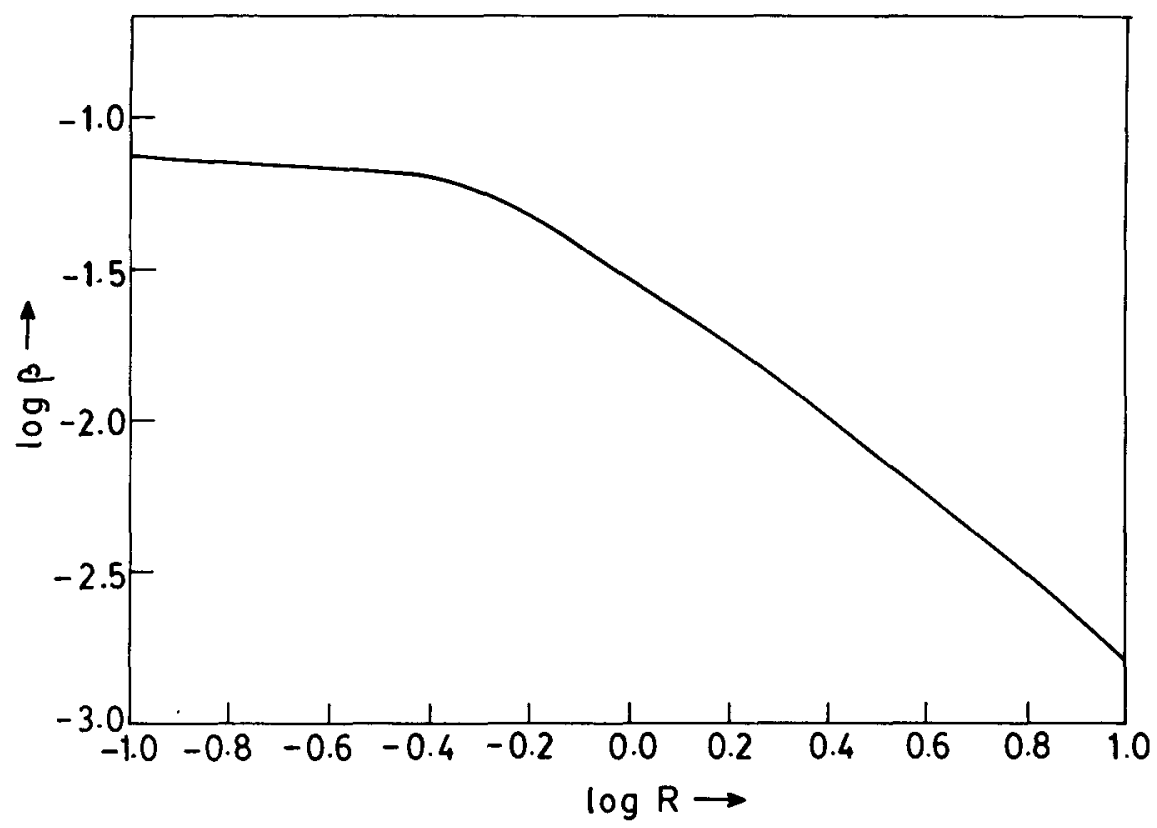

Figure 3. The dependence of $\beta$ on the size of the reactant molecule. $R$ is equal to $2 a / \sigma$ where $a$ is the radius of the reactant molecule. The values of the dielectric constant and the reduced density of the solvent are 18.0 and 0.8 , respectively.

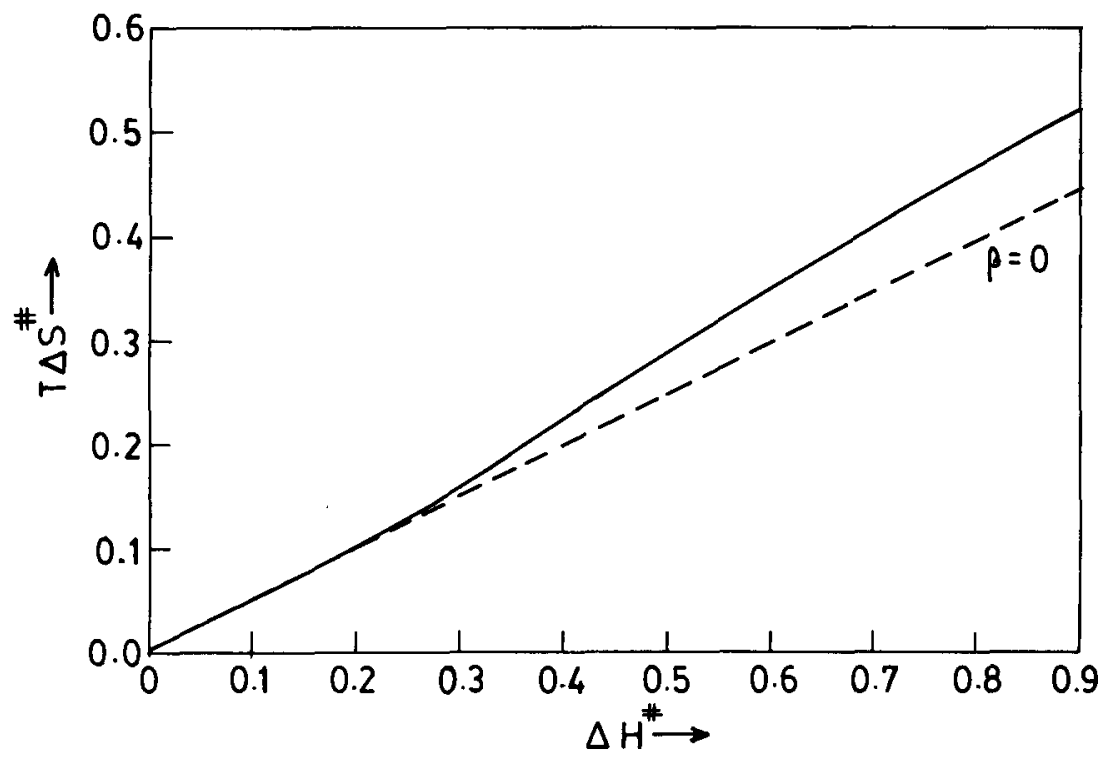

Figure 4. The plot of the entropy of activation $\left(T \Delta S^{\#}\right)$ against the enthalpy of activation calculated for different values of the reactant-solvent molecular size ratio, $R$. The solid curve shows the calculated values and the dashed one is for $\beta=0$. The values of the different parameters are the same as in figure 3. 
becomes very small and in that limit the linear relation can again hold with $\beta \simeq 0$. This behaviour is clear in figure 4 where $T \Delta S^{\#}$ is plotted against $\Delta H^{\#}$ calculated for different sizes of the reactant molecule. Also note that this behaviour is in agreement with the continuum model which gives $\beta=0$. It is well-known that continuum models are reliable when the size of the ionic solute is much larger than that of the solvent molecules.

\section{Conclusions}

It is clearly important to understand the validity of the relation given by (2) and also the range of the values of the parameters $\alpha$ and $\beta$. For example, if (2) holds with a $\beta$ which is so small as can be negligible, then (1) simplifies to

$$
k \simeq \frac{k_{B} T}{h} \exp \left[-(1-\alpha) \Delta H^{\sharp} / k_{B} T\right]
$$

Therefore, a reaction may appear to have a significantly lower activation enthalpy than the real value. This result appears to have a special significance in view of several recently studied photoinduced electron transfer reactions that were found to proceed without the intervention of a significant barrier (Barbara and Jarzeba 1988, 1990).

\section{References}

Amis E S 1966 Solvent effects on reaction rates and mechanisms (New York: McMillan)

Bagchi B 1989 Annu. Rev. Phys. Chem. 40115

Bagchi B and Chandra A 1989a J. Chem. Phys. 907338

Bagchi B and Chandra A 1989b Chem. Phys. Lett. 155533

Bagchi B and Chandra A 1991 Adv. Chem. Phys. 801

Barbara P F and Jarzeba W 1988 Acc. Chem. Res. 21195

Barbara P F and Jarzeba W 1990 Adv. Photochem. 151

Bell R P 1937 Trans. Faraday Soc. 33496

Chandra A and Bagchi B 1989a J. Chem. Phys. 901832

Chandra A and Bagchi B 1989b J. Chem. Phys. 912954

Chandra A and Bagchi B 1989c J. Chem. Phys. 913056

Chandra A and Bagchi B 1989d J. Chem. Phys. 917181

Chandra A' and Bagchi B 1989e J. Phys. Chem. 936996

Chandra A and Bagchi B 1990 J. Phys. Chem. 943152

Chandra A and Bagchi B 1991 J. Chem. Phys. 942258

Entelis S G and Tiger R P 1976 Reaction kinetics in the liquid phase (New York: Wiley)

Evans and Polanyi M 1936 Trans. Faraday Soc. 321333

Fleming G R and Wolynes P G 1990 Physics Today 4336

Frohlich H 1958 Theory of dielectrics (Oxford: University Press)

Glasstone S, Laidler K J and Eyring H 1941 The theory of rate processes (New York: McGraw Hill)

Grey C G and Gubbins K E 1984 Theory of molecular fluids (Oxford: Clarendon) vol. I

Hansen J P and McDonald I R 1986 Theory of simple liquids (London: Academic Press)

Hynes J T 1985 Annu. Rev. Phys. Chem. 36573

Kosower E M and Huppert D 1986 Annu. Rev. Phys. Chem. 37127

Marcus R 1956 J. Chem. Phys. 966979

Marcus R 1964 Annu. Rev. Phys. Chem. 15155

Marroncelli M, McInnis J and Fleming G R 1989 Science 2431674

McMannis G E and Weaver M J 1989 J. Chem. Phys. 90 912, 1720 
Neuman M 1986 Mol. Phys. 5797

Onsager L $1953 \mathrm{~J}$. Am. Chem. Soc. 581485

Raineri F O, Zhou Y, Friedman H L and Stell G 1991 Chem. Phys. (in press)

Reichardt C 1979 Solvent effects in organic chemistry (New York: Verlag Chemie)

Simon J D 1988 Acc. Chem. Res. 21128

Wei D and Patey G N 1990 J. Chem. Phys. 931399

Wertheim M S 1971 J. Chem. Phys. 554291

Wynne-Jones W F K and Eyring H 1935 J. Chem. Phys. 3492 